\title{
Bullectomy: A Waste of Space or Room for Improvement?
}

\author{
Samuel V. Kemp ${ }^{a}$ Felix J.F. Herth ${ }^{c}$ Pallav L. Shah ${ }^{a, b}$ \\ ${ }^{a}$ The National Institute for Health Research Unit, Royal Brompton and Harefield NHS Foundation Trust, and Imperial \\ College, and ${ }^{b}$ Chelsea and Westminster Hospital NHS Foundation Trust, London, UK; ' ${ }^{c}$ Department of Pneumology \\ and Critical Care Medicine, Thoraxklinik, Translational Lung Research Center Heidelberg (TLRCH), Member of the \\ German Lung Research Foundation (DZL), University of Heidelberg, Heidelberg, Germany
}

There has been a resurgence of interest in lung volume reduction therapies over the last few years following the development of endobronchial techniques as an alternative to surgery, with the recent publication of a number of randomised controlled trials [1-3]. Undoubtedly, if patients are appropriately selected, clinically (and statistically) meaningful benefits can be obtained in an increasingly broad group of patients with severe emphysema, although refinement of selection criteria for the individual techniques is still ongoing. The greatest evidence of benefit currently exists for those with heterogeneous emphysema, although the data from the IMPACT trial [4] of valves in homogeneous disease are due to be released in the not too distant future.

Although the aetiology may be subtly different, giant bullous disease could be seen as heterogeneous disease taken to its extreme, and surgery for giant bullous disease has been practiced successfully for several decades. Strange therefore that there is so little in the literature on the treatment of this distinct patient group, particularly as results following surgical bullectomy can be spectacular and genuinely life-changing in individual patients, with evidence to show persistence of benefit out to at least 5 years [5]. Figures for the UK, however, show an average of fewer than 200 procedures a year across 3 annual audits from 2011-2012 to 2013-2014 [6], with one major unit reporting only 20 such procedures over a 12 -year period [7].

\section{KARGER}

E-Mail karger@karger.com www.karger.com/res
In addition, there are no universally accepted criteria for intervention, and the literature is in very large part restricted to case series of surgical treatments, with no comparison of different techniques for achieving decompression or excision. Not everybody either wants or is fit for surgical intervention, and, perhaps slightly surprisingly, the interventional pulmonology community has been a little late to the table. This is probably due to the received wisdom that surgical treatment of giant bullae gives universally excellent results, but what evidence there is does not necessarily support this view even in stable patients [5].

Two case reports in this journal highlight the varied approach to the management of giant bullae and the lifechanging (or saving) results that can be achieved following intervention in these patients. In the first study, Goud et al. [8] report a case of emergency decompression of a rapidly expanding giant bulla in a ventilated patient using a combination of endobronchial valves and transthoracic needle decompression. Definitive surgical management was deemed inappropriate and very high risk, and this novel approach undoubtedly saved the life of a very sick patient. In the foreword to his recent text on diseases of the large airways, Mehta [9] muses that a good bronchoscopist is the one who knows when not to perform a procedure, and some readers will ask 'why use 2 techniques where 1 will do?'. Indeed, either technique may well have resulted in decompression of the bulla. However, con-
(C) 2016 S. Karger AG, Basel

0025-7931/16/0924-0218\$39.50/0
Dr. Samuel V. Kemp

Royal Brompton Hospital Sydney Street

London, SW3 6NP (UK)

E-Mail s.kemp@ rbht.nhs.uk 
cerns over post-procedure air leak with transthoracic needle aspiration or gradual/incomplete decompression with intra-bronchial valves, either of which could have had catastrophic consequences for the patient, meant that a combination of approaches was required, and the rapid clinical improvement vindicates that decision.

The second report [10] presents 3-year follow-up data from a patient who underwent bronchoscopic autologous blood installation as part of a previously published clinical trial [11]. Adapting a technique first published by Kanoh et al. [12], this approach uses the injection of $240 \mathrm{ml}$ of autologous blood via an extended working channel directly into a bullous space under fluoroscopic screening at bronchoscopy. At present, the blood is collected into sterile syringes in $60-\mathrm{ml}$ aliquots and immediately injected into the bullous space. Kanoh's technique only instilled $5-10 \mathrm{ml}$ of blood, using blood as a carrier to instill thrombin. The exact mechanism of action is not clear, but is probably a combination of inflammatory reaction, obliteration of small ventilatory channels, and other as yet undefined processes. The patient continues to demonstrate improvements in spirometry, lung volumes, and breathlessness, with the persistence of almost complete collapse of the treated left upper lobe bulla. The effects following autologous blood instillation are slower than those of other bronchoscopic techniques, and it may take more than 3 months before objective changes in the size of the bullae and patient symptoms are observed. Understanding the mechanism of action may help refine the process to achieve more consistent results and perhaps a more rapid effect.

It is this ability to innovate and adapt that characterises the interventional pulmonology community, and the authors are to be commended on their novel approaches. However, in an era of rapidly expanding interventional pulmonology services and a renaissance in lung volume reduction therapies, giant bullous disease has been left behind somewhat, and the authors have only been able to locate one study registered on a clinical trials database dedicated to the treatment of giant bullous emphysema not related to pneumothorax (Bronchoscopic Intrabullous Autologous Blood Instillation (BIABI) for Emphysema, ClinicalTrials.gov Identifier: NCT01727037). The recent legalisation of cannabis for medicinal (and, in some cases, recreational) use in a number of states in the USA, and its predilection for causing bullous disease [13], is also a concern. This is a call to all surgeons and interventional pulmonologists with an interest in the treatment of those with emphysema to focus even a small amount of their attention on this neglected disease - to develop innovative new techniques, robustly test the evidence for existing techniques, compare surgical and nonsurgical treatments, and expand the eligibility criteria - so that we can finally offer patients evidence-based advice and a choice of therapies with proven benefit.

\section{References}

$>_{1}$ Klooster K, ten Hacken NH, Hartman JE, Kerstjens HA, van Rikxoort EM, Slebos DJ: Endobronchial valves for emphysema without interlobar collateral ventilation. N Engl J Med 2015;373:2325-2335.

- 2 Sciurba FC, Criner GJ, Strange C, Shah PL, Michaud G, Connolly TA, Deslée G, Tillis WP, Delage A, Marquette CH, Krishna G, Kalhan R, Ferguson JS, Jantz M, Maldonado F, McKenna R, Majid A, Rai N, Gay S, Dransfield MT, Angel L, Maxfield R, Hertz FJ, Wahidi MM, Mehta A, Slebos DJ; RENEW Study Research Group: Effect of endobronchial coils vs usual care on exercise tolerance in patients with severe emphysema: the RENEW randomized clinical trial. JAMA 2016;315:21782189.

-3 Herth FJ, Valipour A, Shah PL, Eberhardt R, Grah C, Egan J, Ficker JH, Wagner M, Witt C, Liebers U, Hopkins P, Gesierich W, Phillips M, Stanzel F, McNulty WH, Petermann C, Snell G, Gompelmann D: Segmental volume reduction using thermal vapour ablation in patients with severe emphysema: 6-month re- sults of the multicentre, parallel-group, openlabel, randomised controlled STEP-UP trial. Lancet Respir Med 2016;4:185-193.

4 Improving Patient Outcomes by Selective Implantation of the Zephyr EBV - Study (IMPACT). ClinicalTrials.gov Identifier: NCT02025205.

5 Palla A, Desideri M, Rossi G, Bardi G, Mazzantini D, Mussi A, Giuntini C: Elective surgery for giant bullous emphysema: a 5-year clinical and functional follow-up. Chest 2005; 128:2043-2050.

6 The SCTS Thoracic Surgery Audit Group: The Thoracic Surgery Registry Brief Report Audit Years 2011-2012 to 2013-2014. Society for Cardiothoracic Surgery in Great Britain and Ireland.

7 Clark SJ, Zoumot Z, Bamsey O, Polkey MI, Dusmet M, Lim E, Jordan S, Hopkinson NS: Surgical approaches for lung volume reduction in emphysema. Clin Med (Lond) 2014; 14:122-127.

8 Goud A, Krimsky W, Caldwel M, Perry B, Heiraty P, Sarkar S, Harley DP, Selinger S:
Percutaneous bullectomy in conjunction with endobronchial valve placement as an alternative to surgical management of giant bullae. Respiration 2016;91:523-526.

9 Metha AC, Jain P, Gildea TR: Diseases of the Central Airways: A Clinical Guide. Cham, Springer International Publishing, 2016.

10 Kemp SV, Zoumot Z, Shah PL: Three-year follow-up of a patient with a giant bulla treated by bronchoscopic intrabullous autologous blood instillation. Respiration 2016; 92:283-284.

11 Zoumot Z, Kemp SV, Caneja C, Singh S, Shah PL: Bronchoscopic intrabullous autologous blood instillation: a novel approach for the treatment of giant bullae. Ann Thorac Surg 2013;96:1488-1491.

12 Kanoh S, Kobayashi H, Motoyoshi K: Intrabullous blood injection for lung volume reduction. Thorax 2008;63:564-565.

13 Johnson MK, Smith RP, Morrison D, Laszlo G, White RJ: Large lung bullae in marijuana smokers. Thorax 2000;55:340-342.
Bullectomy: A Waste of Space or Room for Improvement? 